PROCEEDINGS OF THE

AMERICAN MATHEMATICAL SOCIETY

Volume 130, Number 9, Pages 2771-2775

S 0002-9939(02)06385-2

Article electronically published on February 12, 2002

\title{
ON POSSIBLE NON-HOMEOMORPHIC SUBSTRUCTURES OF THE REAL LINE
}

\author{
P. D. WELCH \\ (Communicated by Carl G. Jockusch, Jr.)
}

\begin{abstract}
We consider the problem, raised by Kunen and Tall, of whether the real continuum can have non-homeomorphic versions in different submodels of the universe of all sets. This requires large cardinals, and we obtain an exact consistency strength:

Theorem 1. The following are equiconsistent:

(i) $Z F C+\exists \kappa$ a Jónsson cardinal;

(ii) $Z F C+\exists M$ a sufficiently elementary submodel of the universe of sets with $\mathbb{R}_{M}$ not homeomorphic to $\mathbb{R}$.

The reverse direction is a corollary to:

Theorem 2. $\mathfrak{c}$ is Jónsson $\Longleftrightarrow \exists M \prec H\left(\mathfrak{c}^{+}\right) \exists X_{M}$ hereditarily separable, hereditarily Lindelöf, $T_{3}$ with $X \neq X_{M}$.

We further consider the large cardinal consequences of the existence of a topological space with a proper substructure homeomorphic to Baire space.
\end{abstract}

\section{INTRODUCTION}

Tall in 8] and Junqueira \& Tall in [3] consider the question of the behaviour of a topological space $X=\langle X, T\rangle$ when $X \in M$, where $M$ is a submodel, not necessarily transitive, of the universe of all sets. The topology they consider for $X_{M}$ is that generated by the open sets of $T$ that happen to be in $M$. Thus $X_{M}=\left\langle X \cap M, T_{M}\right\rangle$, where $T_{M}$ is that generated by $\{U \cap M \mid U \in T \cap M\}$. In general this will not coincide with the usual relative topology. We refer to this $\left\langle X_{M}, T_{M}\right\rangle$, somewhat incorrectly, as a "substructure" of $\langle X, T\rangle$. (In fact, as they observe, it suffices to take $M$ a submodel of the class $H(\theta)$ of sets of hereditary cardinality $\theta$, where $\langle X, T\rangle \in H(\theta)$.) We refer the reader to these papers for further discussion and motivation of these ideas. The purpose of this note is to be brief, and state a theorem that characterises Jónsson cardinals:

Definition 1.1. (i) A Jónsson algebra $\mathcal{A}=\left\langle A,\left\langle f_{n}\right\rangle_{n \in \omega}\right\rangle$ is an algebra on $A$ of finitary functions $f_{n}:[A]^{n} \longrightarrow A$ that has no proper subalgebra of the same cardinality as that of $A$.

(ii) A cardinal $\kappa$ is a Jónsson cardinal if there is no Jónsson algebra $\mathcal{A}$ with $\kappa \subseteq A$.

Received by the editors January 16, 2001 and, in revised form, March 27, 2001.

2000 Mathematics Subject Classification. Primary 54A05, 03E35, 03E02, 54A35, 03E55; Secondary 54B05.

Key words and phrases. Real continuum, subspaces, Jónsson cardinals.

(C)2002 American Mathematical Society 
In the first part of this note we prove the theorems of the abstract, which involve this concept. The reader is assumed familiar with, or to have access to a copy of [6] and [8]. Our definitions and terminology are standard. In the final part of the paper we look at a question of Tall's from [8] concerning the possibility of proper topological substructures being homeomorphic to the irrationals. The "large cardinal" theorem there has a proof that presupposes some basic familiarity with core model theory.

It is known that the existence of a Jónsson cardinal has mild large cardinal strength (Ramsey cardinals are Jónsson, and so their strength is less than that of a measurable cardinal). One may prove outright in $Z F C$ that no $\aleph_{n}(n<\omega)$ is Jónsson. It is not known whether $\aleph_{\omega}$ may be Jónsson, although it is known it would require very large cardinals to render this consistent. The first candidate for the continuum $\mathfrak{c}$ to be Jónsson is $\aleph_{\omega_{1}}$. (Simply because $c f(\mathfrak{c})>\omega$ and no regular cardinal below $\aleph_{\omega_{1}}$ is Jónsson by results of Shelah [7, Woodin, and Tryba [9].)

\section{JónsSON CARDINALS AND THE CONTINUUM}

The following (Theorem 2 of the abstract) should be compared to Theorem 4 of [8].

Theorem 2.1. $\mathfrak{c}$ is Jónsson $\Longleftrightarrow \exists M \prec H\left(\mathfrak{c}^{+}\right) \exists X_{M}$ hereditarily separable, hereditarily Lindelöf, $T_{3}$ with $X \neq X_{M}$

Proof. $(\Rightarrow)$ Let $\mathfrak{c}$ be Jónsson. Then $\exists M \prec\left\langle H_{\mathfrak{c}^{+}}, \in\right\rangle$ with $\mathfrak{c} \in M,|M \cap \mathfrak{c}|=\mathfrak{c}, M \cap \mathfrak{c} \neq$ c. That such an $M$ with these properties exists is a standard fact about Jónsson cardinals; see e.g. [4], $\S 8$. As $H_{\mathfrak{c}^{+}} \models \exists f: \mathbb{R} \longleftrightarrow \mathfrak{c} \in O n$, it follows that $\mathbb{R} \cap M \neq \mathbb{R}$. However, as [3] shows, for the case of first countable spaces $X$, we have that in fact $X \cap M=X_{M}$. Hence $\mathbb{R}_{M} \neq \mathbb{R}$.

$(\Leftarrow)$ Suppose $\mathfrak{c}$ is not Jónsson. It suffices by the proof of [8], Theorem 4 , to show that $[0,1] \subseteq M$. For this it is enough to show $\mathfrak{c} \subseteq M$. But $H_{c^{+}} \models " ~ \exists\left\langle\mathfrak{c}, f_{n}\right\rangle_{n<\omega}$ a Jónsson algebra on $\mathfrak{c}$ ". Hence $M$ is a model of the above sentence. Let $\left\langle\mathfrak{c}, g_{n}\right\rangle_{n<\omega} \in$ $M$ be a witness to this. (It is unproblematic to assume that the field of the algebra is $\mathfrak{c}$ itself.) Then each $g_{n} \in M$, and, if $g_{n}:[\mathfrak{c}]^{n} \longrightarrow \mathfrak{c},\left\langle M \cap \mathfrak{c}, g_{n}\left\lceil[M \cap \mathfrak{c}]^{n}\right\rangle_{n<\omega}\right.$ is a subalgebra of $\left\langle\mathfrak{c}, g_{n}\right\rangle_{n<\omega}$. By definition of $\left\langle g_{n}\right\rangle$ we have $M \cap \mathfrak{c}=\mathfrak{c}$.

Corollary 2.2. If $\mathfrak{c}$ is not Jónsson, and if $X_{M}$ is an uncountable complete separable metric space, then $X_{M}=X$.

Corollary 2.3. If $\mathfrak{c}$ is not Jónsson, and $X_{M}$ is homeomorphic to an uncountable Borel subspace of $\mathbb{R}$, then $X_{M}=X$.

In 8] already some anti-large cardinal assumption was used. In the former corollary the weaker hypothesis "c is not Jónsson" replaces " $\mathrm{CH}+\neg 0^{\#}$ " of [8], Theorem 21 a), and just " $\neg 0 \#$ " of Cor. 25., op.cit. The proofs are the same.

In [6] Kunen and Tall asked:

Question: What is a lower bound on the consistency strength of:

$$
\exists 2^{\mathfrak{c}} \text {-many non-homeomorphic } \mathbb{R}_{M} \text { of size } \mathfrak{c} \text { ? }
$$

They show ([6], Theorems 16 and 8, respectively):

(i) $\operatorname{Con}(Z F C+\exists$ a Ramsey cardinal $) \Rightarrow \operatorname{Con}(Z F C+(*))$.

(ii) $Z F C+\exists \mathbb{R}_{M}\left(\left|\mathbb{R}_{M}\right|=\mathfrak{c} \wedge \mathbb{R}_{M}\right.$ is not homeomorphic to $\left.\mathbb{R}\right) \vdash 0^{\#}$ exists.

We raise this latter lower bound to that of a Jónsson cardinal. 
Theorem 2.4. $Z F C+\exists \mathbb{R}_{M}\left(\left|\mathbb{R}_{M}\right|=\mathfrak{c} \wedge \mathbb{R}_{M}\right.$ is not homeomorphic to $\left.\mathbb{R}\right) \vdash \mathfrak{c}$ is a Jónsson cardinal

Proof. This is just the argument of $(\Leftarrow)$ of Theorem 2.1

In their model of $(i)$ in which $(*)$ holds, $\mathfrak{c}$ has been made equal to the Ramsey cardinal $\kappa$ of the ground model by c.c.c. forcing. In such a model $\kappa$ is Jónsson (see [1]). As Jónsson cardinals are equiconsistent with Ramseys, this shows that the authors of [6] were using exactly the right hypothesis. In their model $\mathfrak{c}$ is weakly inaccessible.

Their proof that there were at least $2^{\mathfrak{c}}$ non-homeomorphic $\mathbb{R}_{M}$ amongst their $2^{\mathfrak{c}}$ many different submodels $M$ used Lavrentieff's theorem on extending putative homeomorphisms to $G_{\delta}$ 's and a counting argument. We next observe that in their construction all the $\mathbb{R}_{M}$ are non-homeomorphic-again using Lavrentieff, but using a further property of indiscernibles arising from Ramsey cardinals:

Definition 2.1. Let $\mathcal{A}=\left\langle L_{\kappa}[A], A, \vec{R}, \vec{f}, \ldots\right\rangle$ be a first order structure with $\kappa \subseteq$ $|\mathcal{A}| . I \subseteq \kappa$ is a good set of indiscernibles for $\mathcal{A}$ if for all $\gamma \in I$

(i) $\left\langle L_{\gamma}[A \cap \gamma], \in, A \cap \gamma, \vec{R} \cap \gamma, \vec{f}\lceil\gamma, \ldots\rangle \prec \mathcal{A}\right.$;

(ii) $I \backslash \gamma$ is a set of indiscernibles for the structure $\left\langle L_{\delta}[A], \in, A, \vec{R}, \vec{f}, \ldots\langle\xi\rangle_{\xi \leq \gamma}\right\rangle$.

Fact 2.1. Suppose $\kappa$ is a Ramsey cardinal. Then for evey first order structure $\mathcal{A}$ as above there is a good sequence of indiscernibles of order type $\kappa$. (See, e.g., [2], Ch. 16.)

Using this and the notation of $[6$, Theorem 16, if $I$ is decomposed into a sequence $\left\langle I_{\alpha} \mid \alpha<\kappa\right\rangle$ of mutually disjoint subsets each of size $\kappa$, then each $M_{\alpha}={ }_{d f}\left\{\tau_{G} \mid \tau\right.$ is a $\mathbb{P}$-name $\left.\tau \in \mathcal{H}\left(I_{\alpha}\right)\right\}$ has a version of $\mathbb{R}: \mathbb{R}_{M}$. (We are taking here $\mathcal{H}(Y)$ to be defined as the Skolem hull of $Y$ in $H\left(\lambda^{+}\right)$.) We have then:

Lemma 2.5. The collection of $\mathbb{R}_{\alpha}={ }_{d f} \mathbb{R}_{M_{\alpha}}$ are all pairwise non-homeomorphic.

Proof. As Kunen and Tall argue, by Lavrentieff's theorem, if $\mathbb{R}_{\alpha}$ were homeomorphic with $\mathbb{R}_{\beta}$ via a homoeomorphism $g$ say, $g$ could be extended to a homeomorphism between two $G_{\delta}$ subsets of $\mathbb{R} ; \tilde{g}: G_{\alpha} \longrightarrow G_{\beta}$. But such a homeomorphism, and $G_{\alpha}, G_{\beta}$, are essentially coded by a real, $r$ say. But any real of $V[G]$ (where $G$ is the generic for the c.c.c. forcing $\mathbb{P}$ adding $\kappa$ many reals) is added at some initial stage of the forcing. We may thus factor $\mathbb{P}$ as $\mathbb{P}_{0} * \mathbb{P}_{1}$ with $r$ being added by $G_{0}={ }_{d f} G \cap \mathbb{P}_{0}$. Let $\dot{r}$ be a $\mathbb{P}$-name (and a $\mathbb{P}_{0}$-name) for $r$. But for all sufficiently large $\gamma$, the sets $I_{\gamma}$ are good indiscernibles for $\left\langle L_{\kappa}[A], \in, \mathbb{P}, \Vdash_{\mathbb{P}}, \mathbb{P}_{0}, \Vdash_{\mathbb{P}_{0}}, \dot{r},\langle\xi\rangle_{\xi<\gamma}\right\rangle$, where $\mathbb{P}_{0}, \dot{r} \in V_{\gamma}$ and $A \subseteq \kappa$ is assumed to code up $V_{\kappa}$ so that for any strong inaccessible $\eta<\kappa$ we have $V_{\eta}=L_{\eta}[A \cap \eta]$. They are thus easily seen to be good indiscernibles in $V\left[G_{0}\right]$. Now the argument finishes as in [6]: let $f: \kappa \leftrightarrow \mathbb{R}$ be a bijection in $V[G]$ named by a term in $\mathcal{H}(\varnothing)$; if $i>\gamma$ is any indiscernible in $I_{\beta} \backslash I_{\alpha}$, we have $f(i) \in \mathbb{R}_{\beta} \backslash \mathbb{R}_{\alpha}$. But then $\tilde{g}^{-1}(f(i)) \in \mathbb{R}_{\alpha}$, and thus $i \in M_{\alpha}$ although $i$ cannot be named by any term defined from the indiscernibles of $I_{\alpha}$.

To make $\mathfrak{c}$ singular and Jónsson requires the consistency of larger cardinals.

\section{Theorem 2.6.}

$$
\begin{aligned}
\operatorname{Con}(Z F C+\mathfrak{c}>c f(\mathfrak{c})= & \lambda+\exists \mathbb{R}_{M}\left(\left|\mathbb{R}_{M}\right|=\mathfrak{c} \wedge \mathbb{R}_{M} \neq \mathbb{R}\right) \\
\Longleftrightarrow \operatorname{Con}\left(Z F C+\exists\left\langle\kappa_{i} \mid i<\lambda\right\rangle\right. \text { an increasing sequence } & \\
& \text { of measurable cardinals, with } \left.\kappa_{0}>\lambda\right) .
\end{aligned}
$$


Proof (Sketch) $(\Rightarrow)$. The assumption implies again that $\mathfrak{c}$ is Jónsson, by the argument of Theorem [2.1, and that together with the cofinality hypothesis gives an inner model with the sequence of $\lambda$ measurable cardinals by Koepke [5].

$(\Leftarrow)$ Assuming we have measures $\mathcal{U}_{i}$ on $\kappa_{i}$, find a sequence $\left\langle X_{i} \mid i<\lambda\right\rangle$ of measure one sets which are mutually coherently indiscernible, and use the argument of [6]: make $\mathfrak{c}=\sup _{i}\left\{\kappa_{i}\right\}$ by c.c.c. forcing adding reals, and get $2^{\mathfrak{c}}$ many models with different versions of $\mathbb{R}_{M}$.

\section{ON THE POSSIBILITy OF A TOPOLOGICAL SPACE WITH A PROPER SUBSTRUCTURE HOMEOMORPHIC TO BAIRE SPACE}

In [8] an interesting question is raised:

Question: Can there be $\langle X, T\rangle \in M \prec H\left(\lambda^{+}\right)$with $X_{M}$ homeomorphic to the irrationals, but $X_{M} \neq X$ ?

It is easy to see, by the reasoning above, that if the question had a positive answer with $\lambda=\mathfrak{c}$ then $\mathfrak{c}$ would have to be Jónsson (and hence at least $\aleph_{\omega_{1}}$ ). However the possibility exists that $X$ may be of size $\lambda>\mathfrak{c}$, with $|M|=\mathfrak{c}$ and $X_{M}$ homeomorphic to Baire space. This would, prima facie, only imply that $\langle\lambda, \mathfrak{c}\rangle \longrightarrow\langle\mathfrak{c},<\mathfrak{c}\rangle$, a Changconjecture-like property weaker than that of a Jónsson cardinal. I cannot answer the above question, but I can show that large cardinals are still involved.

Theorem 3.1. Suppose the answer to the question is positive, and let $X, T, \lambda, M$ witness this. Then there is an inner model with $\lambda$ a $\mathfrak{c}$-Erdös cardinal.

Proof. We suppose there is no inner model with a measurable cardinal $\kappa \leq \lambda$, for otherwise we have nothing to do: in the core model $K$ below a measurable cardinal every $V$-cardinal $\bar{\lambda} \geq \kappa$ is a Ramsey cardinal, and hence certainly a $\mathfrak{c}$ Erdös cardinal! We show then that in this core model $K \lambda$ satisfies the partition relation $\lambda \longrightarrow(\mathfrak{c})_{2}^{<\omega}$. This suffices. Recall that $\lambda \longrightarrow(\mathfrak{c})_{2}^{<\omega}$ is defined to mean that for every function $F:[\lambda]^{<\omega} \longrightarrow 2$ there is a set $H \subseteq \lambda$ with $|H|=\mathfrak{c}$ and for each $n<\omega$ we have $\left|F^{\text {" }}[H]^{n}\right|=1$, that is, $H$ is homogeneous for $F$. We assume only a slight familiarity with the construction of core models. Such a core model is of the form $K=L[E]$, where $E$ is a sequence of extenders. The point is that, unlike for Gödel's constructible universe $L$, we must have a failure of condensation. Suppose $F \in K$ is a function as above, which is a counterexample to the partition property in $K$. We may assume that $F$ has been chosen least in the natural ordering of $H\left(\lambda^{+}\right)^{K}$ and hence is a definable point in the substructure $M$, as is also the predicate $E\left\lceil\lambda^{\prime}\right.$ coding the whole model's construction up to $\lambda^{\prime}$, the latter a sufficiently large ordinal so that $F \in L_{\lambda^{\prime}}\left[E\left\lceil\lambda^{\prime}\right]\right.$. As $X_{M}$ is assumed homeomorphic to Baire space, $M$ has size $\mathfrak{c} .|M \cap \mathfrak{c}|<\mathfrak{c}$, as otherwise $M$ would contain all of $\mathbb{R}$. Let $\sigma: N \longrightarrow M$ be the inverse of the transitive collapse of $M$, with $N$ transitive. Set $\sigma(\bar{\lambda}, \bar{E}, \bar{F})=\lambda, E, F$. We then note that $O n \cap N \geq \mathfrak{c}$ and $N=$ " $\bar{\lambda}$ is the largest cardinal". Hence $\bar{\lambda} \geq \mathfrak{c}$. The point now is to note that $L_{\mathfrak{c}}[\bar{E}] \neq K_{\mathfrak{c}}={ }_{d f} L_{\mathfrak{c}}[E]$ (the core model constructed up to $\mathfrak{c})$. For if it were, as the least ordinal moved by $\sigma, \operatorname{crit}(\sigma) \leq \delta \leq \mathfrak{c}$ for some regular $\delta$. We should then be able to define an iterable $K$-ultrafilter on an ordinal $\beta<\mathfrak{c}$, and hence an elementary $j: K \longrightarrow K$. This implies there is an inner model with a measurable cardinal, contrary to our global hypothesis. Hence, if $\bar{K}={ }_{d f} K^{N}$, then $\bar{K}_{\mathfrak{c}} \neq K_{\mathfrak{c}}$. If we now perform the comparison iteration of $K$ with $\bar{K}$, we shall see that there is a "mouse" structure $M$ of cardinality $<\mathfrak{c}$ that iterates past $\bar{K}$. If

$$
C=\left\{\kappa_{i} \mid \kappa_{i} \text { is the critical point used at stage } i \text { in the comparison }\right\},
$$


then standard arguments show that an end segment $C_{0} \subseteq C$ is cub in $\mathfrak{c}$ and moreover forms an unbounded set of good indiscernibles for the structure $\left\langle\bar{K}_{\bar{\lambda}}, \in \bar{F}\right\rangle$. Let $D=$ $\sigma$ " $C_{0}$. Then $D$ is a good set of indiscernibles of order type $\mathfrak{c}$ for $\left\langle K_{\lambda}, \in F\right\rangle$. We now appeal to the Jensen Indiscernibles Lemma (see [2], 16.10), and use the uncountable cofinality of $\mathfrak{c}$, to claim that there is $E \supseteq D$ with $E \in K$ and elements of $E$ good indiscernibles for the same structure. But this entails $E$ being a homogeneous set for the function $F$. This contradicts our choice of $F$.

\section{REFERENCES}

[1] K.J.Devlin, Some weak versions of large cardinal axioms in Annals of Mathematical Logic, 5, 1973, 291-325. MR 51:161

[2] A.J.Dodd, The Core Model, London Mathematical Society Lecture Notes in Mathematics Series, 61, CUP, 1982. MR 84a:03062

[3] L.R.Junqueira \& F.D.Tall, The topology of elementary submodels, in Topology 85 its Applications, 82, (1998), 239-266. MR 99a:54003

[4] A. Kanamori, The Higher Infinite, Perspectives in Mathematical Logic, 1994, Springer Verlag, Berlin. MR 96k:03125

[5] P. Koepke, Some applications of short core models, in Annals of Pure ES Applied Logic 37, (1988), 179-204. MR 89a:03092

[6] K.Kunen \& F.D.Tall, The real line in elementary submodels of set theory, in Journal of Symbolic Logic, 65, (2000), 683-691. CMP 2000:15

[7] S. Shelah, Cardinal Arithmetic, Oxford Logic Guides 29, (1994), Oxford University Press, Oxford. MR 96e:03001

[8] F.D.TALL, If it looks and smells like the reals ..., in Fundamenta Mathematicae, 163 No.1, (2000), 1-11. MR 2001d:54003

[9] J. TRYBA, On Jónsson cardinals of uncountable cofinality, in Israel Journal of Mathematics, 49, (1984), 315-324. MR 87b:03118

Department of Mathematics, University of Bristol, Bristol BS8 1TW, England AND - Department Institut Für Formale Logik, WÄhringerstr 25, A-1090 Wien, Austria

E-mail address: welch@logic.univie.ac.at

Current address: Mathematisches Institut, Beringstrasse 6, Bonn, D-53115, Germany 